\title{
Red Light Exposure Delays Appearance and Aroma Deterioration of Fresh-Cut Watermelon during Retail Display
}

\author{
Yue Shi $\mathbb{D}^{1,2}$ Yubin Wang $\mathbb{D D}^{1,3}$ Yue Ma $\mathbb{D}^{2,3}$ Yong Xu $\mathbb{D}^{1,},{ }^{1,4}$ Xiaoyan Zhao $\mathbb{D}{ }^{1,3}$ \\ and Chao Zhang $\mathbb{D}^{1,2}$ \\ ${ }^{1}$ Beijing Academy of Agriculture and Forestry Sciences, Beijing Vegetable Research Center, Beijing 100097, China \\ ${ }^{2}$ Beijing Key Laboratory of Fruits and Vegetable Storage and Processing, Beijing 100097, China \\ ${ }^{3}$ Key Laboratory of Vegetable Postharvest Processing of Ministry of Agriculture and Rural Affaires, Beijing 100097, China \\ ${ }^{4}$ Key Laboratory of Biology and Genetic Improvement of Horticultural Crops (North China) of Ministry of Agriculture and \\ Rural Affaires, Beijing 100097, China
}

Correspondence should be addressed to Chao Zhang; zhangchao@nercv.org

Received 30 March 2020; Revised 28 June 2020; Accepted 16 November 2020; Published 1 December 2020

Academic Editor: Alejandro Hernández

Copyright (C) 2020 Yue Shi et al. This is an open access article distributed under the Creative Commons Attribution License, which permits unrestricted use, distribution, and reproduction in any medium, provided the original work is properly cited.

\begin{abstract}
Appearance and aroma deterioration are the main concerns during the retail display of fresh-cut watermelons. Here, fresh-cut watermelons were exposed to the red, green, blue, and conventional white light at $4^{\circ} \mathrm{C}$ for 4 days, respectively, and their resulting qualities were compared with the conventional white light as the control. Specifically, the red light presented its maximum emissions in $620-650 \mathrm{~nm}$ with a purity of $100 \%$ and an intensity of 1104.7 lux. The red light exposure reduced the weight loss to $1.81 \%$, which was reduced by $51.1 \%$ of the control. The red light exposure reduced the color difference of the fruit surface significantly as well as maintaining its redness. The water-soaking ratio of the red light exposure was also reduced by $62.8 \%$ of the control. Moreover, the red light exposure delayed the aroma deterioration, which resulted from both microbial metabolism and the self-metabolism of volatiles of the fresh-cut watermelon. In summary, the red light exposure was better to delay the appearance and aroma deterioration of fresh-cut watermelons than the conventional white light during their retail display.
\end{abstract}

\section{Introduction}

Fresh-cut watermelon is appreciated all over the world due to its crisp texture and refreshing aroma [1,2]. Crisp texture comes at the cost of fragile cell walls. A break of fragile cell walls usually leads to a water-soaking symptom that presents as tissue translucency, softening, and maceration of the endocarp and placental tissues [3]. This symptom leads the bright redness to a dark redness, thus deteriorating the appearance of the fresh-cut watermelon [4]. Aroma of watermelons is mainly influenced by nonenol/nonenal and their derivatives that are easy to be oxidized or etherified [5-7]. Aroma deterioration occurs once watermelons are cut. Hence, the appearance and aroma deterioration are the main concerns during the retail display of fresh-cut watermelon [8].
Visible light exposure during the retail display is an emerging approach to preserve the quality of fresh-cut fruit and vegetables by inactivating most microorganisms and texture-related enzymes and delaying aroma deterioration [9-11]. In comparison with traditional chemical exposures, visible light exposure takes advantages of nontoxicity, low cost, and free residues [9]. Moreover, visible light exposure behaves as a signaling factor for the photocybernetic function of the plant and simultaneously provides illumination during the retail display $[12,13]$. Consequently, visible light exposure becomes one of the simplest and more environmental friendly ways to preserve the quality of fruit and vegetables $[9,14]$. In view of this, extensive studies have been conducted to investigate the effect of light exposure at various intensities and photoperiods on both the quality and physiology of fresh fruit and vegetables during their retail 
display [15]. Among the studies, blue light exposure $(405 \pm 5 \mathrm{~nm})$ inactivates Salmonella spp. of fresh-cut papaya and mango and maintains their qualities [13, 14]. Visible light exposure (2500 lux) protects fresh-cut romaine lettuce from browning and quality decay [16]. Light exposure $\left(2.4 \times 10^{-5} \mathrm{~mol} \cdot \mathrm{m}^{-2} \cdot \mathrm{s}^{-1}\right)$ inhibits the polyphenol oxidase activity by $26 \%$ and peroxidase by $16 \%$ of fresh cauliflower and lowers the browning index by $33 \%$ [11]. Moreover, the intermittent visible light exposure $\left(5.0 \times 10^{-5} \mathrm{~mol} \cdot \mathrm{m}^{-2} \cdot \mathrm{s}^{-1}\right)$ decreases browning and reduces moisture loss of fresh-cut lettuces compared to continuous light exposure [17]. However, the intense light pulse exposure $\left(1.2 \times 10^{5} \mathrm{~J} \cdot \mathrm{m}^{-2}\right)$ presents a negative impact on qualities of fresh-cut watermelon [18].

Retail display occupied the main shelf life of fresh-cut watermelons, and thereby conditions of retail display were an important segment to affect the qualities of fresh-cut watermelons [19-23]. Our recently reported manuscript showed that the visible light exposure of 3000 lux reduces the water-soaking symptom of fresh-cut watermelon by $18.3 \%$ [8]. The conventional white light is composed of red, green, blue, and some other wavelength spectrals. Hence, the aim of this study was to explore the effect of light spectral on the appearance and aroma of fresh-cut watermelons. The freshcut watermelons were exposed to white, red, green, and blue light for 4 days, respectively, and their resulting qualities were compared with the white light as the control.

\section{Materials and Methods}

2.1. Exposure Treatment. Seedless watermelons (Citrullus vulgaris, var. Guomin No.2) were mature and uniform, being harvested in June of 2016 and 2017 from Beijing Sijiqing Farm (Sijiqing Farm, Haidian District, Beijing). The fruits were round with regular stripes and weighed about $3 \sim 5 \mathrm{~kg}$ per fruit. The flesh of the fruits was red and crispy with a soluble solid content of $9.0 \% \sim 11.5 \%$.

Nine fruits were stored at $4^{\circ} \mathrm{C}$ for $24 \mathrm{~h}$ before cutting. The surface of the fruits was washed in icy sodium hypochlorite solution $\left(100 \mathrm{mg} \cdot \mathrm{L}^{-1}\right)$ and was flushed twice with icy water, and then the fruits were moved to a sanitary processing room with a temperature of $4^{\circ} \mathrm{C}$. The fruits were peeled until the red flesh was revealed. The flesh was cut into cubes with the dimensions $0.025 \mathrm{~m} \times 0.025 \mathrm{~m} \times 0.025 \mathrm{~m}$. All cubes were mixed, and the random 12 pieces of cubes weighing approximately $0.28 \mathrm{~kg}$ were fitted in a polyethylene tray and sealed with a transparent cover. The trays were stored in a 4shelf double-door cabinet at $4^{\circ} \mathrm{C}$ for 4 days. The cabinet was illuminated by while, red, green, and blue lamps (T8 LED lamp with a length of $1.2 \mathrm{~m}, 18 \mathrm{~W}$, Zhongshan Blue Shark Lighting Co., Ltd. Zhongshan, China) in the top of each layer during the entire retail display. The distance between the lamp and the sample was $13.5 \mathrm{~cm}$. The samples before the storage were nominated as fresh, and the white exposure treatment was the control.

In each treatment, eight trays were stored randomly. After stored for 4 days, three trays were for the weight loss measurements, one tray was for the color and water-soaking ratio measurements, one tray was for the soluble solid content, titratable acid, and Vc content measurements, one tray was for the firmness measurements, one tray was for the antioxidant capacity measurements, and one tray was for the aroma comparison.

2.2. Characteristic of LED Lamp. The illumination intensity of the LED lamps was monitored by a portable light meter (TES-1339R Data Logger, TES Electrical Electronic Corp., Taipei, China). The spectral characteristic of the LED lamps was monitored by an optical radio meter (CL500A, Konica Minoltas Co. Ltd, Japan). Specially, the sensors of two meters were placed in a covered tray at the same level of the sample. The illumination intensity was the average of six measurements in the different points of the shelf.

The color of illumination is expressed in the $Y x y$ space, where $Y$ indicates luminance and $x$ and $y$ are the chromaticity values calculated from the three values of $\mathrm{XYZ}$ via equation (1). The purity of illumination is the distance between white dots and samples divided by the distance between white dots and spectral points:

$$
\begin{aligned}
& x=\frac{X}{X+Y+Z}, \\
& y=\frac{Y}{X+Y+Z},
\end{aligned}
$$

where $X, Y$, and $Z$ are the absolute values of chromatic coordinates $R, G$, and $B$ of the sample in the CIE-RGB system.

The intensities of the red, green, and blue light were $(1104.7+55.7),(2977+181.5)$, and $(155.1+15.3)$ lux, respectively. Their maximum emissions were located in 620-650, 510-530, and 440-460 nm, respectively (Figure 1). The red and blue lights were located in the main wavelength track of 638 and $447 \mathrm{~nm}$ with a purity of $100 \%$ in the Herm Holtz profile, respectively, while the green light presented a complementary wavelength of $520 \mathrm{~nm}$ with a purity of $86.4 \%$. The red, green, and blue light exposures provided appropriate illumination for the fresh-cut watermelon.

The white light was located in the blackbody track and presented daylight spectral characteristics with an intensity of $(2939.7+155.6)$ lux. Its maximum emission overlapped with the spectral of the red, green, and blue lights in 430-460 and $500-640 \mathrm{~nm}$.

\subsection{Determination of Weight Loss, Soluble Solid Content, and} Titratable Acid. The weight loss ratio was the percentage of the weight difference between the initial and final weight in the initial weight.

Soluble solid content of the sample was measured via a pocket refractometer (Pal- $\alpha$, ATAGO Co., Ltd., Japan) with distilled water as a blank.

An aliquot of $10 \mathrm{~mL}$ juice was titrated automatically with a standardized $0.01 \mathrm{~mol} \cdot \mathrm{L}^{-1} \mathrm{NaOH}$ to the end point of $\mathrm{pH} 8.1$ by a $\mathrm{pH}$ meter (TitroLine ${ }^{\circledR}$-SI Analytics $\mathrm{GmbH}$, Mainz, Germany). The volume of $\mathrm{NaOH}$ (by multiplying with 0.67 ) was converted to mg malic acid per milliliter juice. 


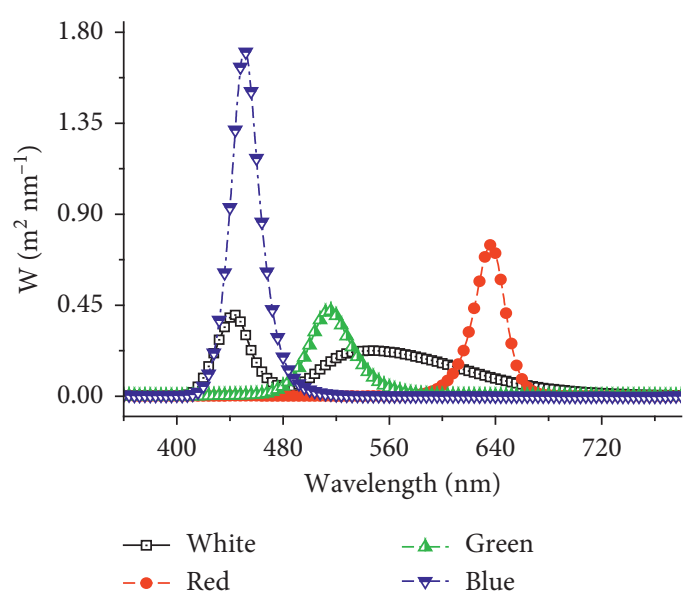

(a)

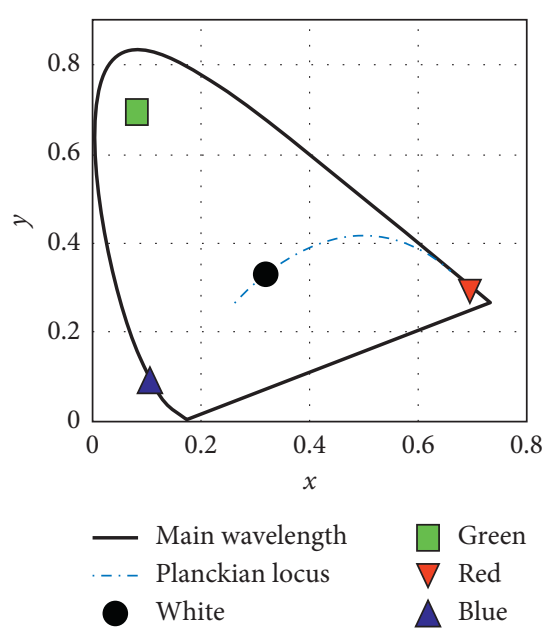

(b)

FIgURE 1: Spectral distribution (a) and the Herm Holtz (b) profiles of LED lamps.

2.4. Determination of Vc Content. The Vc content was determined via a recently reported HPLC method [24]. An aliquot of $25 \mathrm{~mL}$ of the juice was mixed with $25 \mathrm{~mL}$ of a solution containing $45 \mathrm{~g} \cdot \mathrm{L}^{-1}$ of metaphosphoric acid and $7.2 \mathrm{~g} \cdot \mathrm{L}^{-1}$ of dithiothreitol. The mixture was centrifuged at $20,000 \times \mathrm{g}$ for $15 \mathrm{~min}$ at $4^{\circ} \mathrm{C}$, and the supernatant was vacuum filtered through the Whatman No. 1 paper. The sample was then passed through a millipore $0.45 \mu \mathrm{m}$ membrane into an opaque vial and kept at $-80^{\circ} \mathrm{C}$ until being used. An aliquot of $20 \mu \mathrm{L}$ was injected into Agilent 1200 series HPLC (Agilent Technologies, Palo Alto, California) fitted with a reversephase C18 Spherisorb ${ }^{\circledR}$ ODS2 $(5 \mu \mathrm{m})$ stainless-steel column $(4.6 \mathrm{~mm} \times 250 \mathrm{~mm})$. The mobile phase was a $0.01 \%$ sulphuric acid solution adjusted to $\mathrm{pH}$ 2.6. The flow rate was fixed at $1 \mathrm{~mL} \cdot \mathrm{min}^{-1}$ and monitored at $245 \mathrm{~nm}$ at $25^{\circ} \mathrm{C}$. Vc content was quantified via a calibration curve based on ascorbic acid pure standards, and results were expressed as relative Vc concentration.

2.5. Determination of Firmness. The fruit cube was placed on the test platform of a TA.XT2i plus texture analyzer (Stable Micro Systems Ltd., Godalming, Surrey, UK). The P/5 trigger was compressed to $50 \%$ of the total height with a pretest speed of $10 \mathrm{~mm} \cdot \mathrm{s}^{-1}$, test speed of $2 \mathrm{~mm} \cdot \mathrm{s}^{-1}$, and posttest speed of $10 \mathrm{~mm} \cdot \mathrm{s}^{-1}$. During the compression, the force of the trigger increased quickly and then vibrated in a small range at a certain level. The time of the vibration was defined as the statistical interval. The average force of the statistical interval was defined as the firmness of fruit cubes.

2.6. Determination of Color and Water-Soaking Ratio. Color $L^{a *}, a^{a *}$, and $b^{a *}$ values were measured in a reflectance mode six times at $23^{\circ} \mathrm{C}$ (Chromameter CR-300, Minolta, Japan). The color difference $(\Delta E)$ was calculated by the following equation:

$$
\Delta E=\sqrt{\left(L_{1}-L_{0}\right)^{2}+\left(a_{1}-a_{0}\right)^{2}+\left(b_{1}-b_{0}\right)^{2}},
$$

where $\Delta E$ is the color difference between a sample and the control; $L_{1}$ and $L_{0}$ are the lightness of a sample and the fresh one, respectively; $a_{1}$ and $a_{0}$ are the redness of a sample and the fresh one, respectively; and $b_{1}$ and $b_{0}$ are the yellowness of a sample and the fresh one, respectively.

The samples were photographed with uniform exposure parameters via Canon EOS600D (Canon Group, Japan). The image was calculated by Image-Pro Plus (Version 6.0, Media Cybernetics, Inc., Bethesda, MD). The pixels with the red index of 140 190 in the RGB system were selected as the water-soaking region and were marked in yellow. The watersoaking ratio was counted as the number of yellow pixels in the total fruit area. The water-soaking ratio was an average of more than six replicates.

2.7. Measurement of DPPH Radical Scavenging Capacity and ORAC Assay. The upper and lower surfaces of the sample were used for DPPH radical scavenging capacity and ORAC measurements. Specifically, the upper/lower surface of the sample was cut with a thickness of $0.3 \mathrm{~cm}$ from the surface, nominated as exposed/unexposed. The mixed fruit chips were homogenized in a Philips food mixer (HR1861 mixer, Philips, Dongguan, China) and centrifuged at $7000 \times \mathrm{g}$ and $4^{\circ} \mathrm{C}$ for $10 \mathrm{~min}$ (Sigma 3-18K, Sartorius, Gottingen, Germany). The supernatant was evaluated for the DPPH radical scavenging capacity and ORAC value.

Specifically, the free radical scavenging capacity was evaluated using the stable 2,2-diphenyl-1-picryhydrazyl (DPPH) radical [25]. The final concentration was $1 \times 10^{-4} \mathrm{~mol} \cdot \mathrm{L}^{-1}$ for DPPH with butylated hydroxytoluene as the control. The DPPH radical scavenging activity was calculated as a percentage of DPPH discoloration and as butylated hydroxytoluene equivalent.

The oxygen radical absorbance capacity (ORAC) of the sample was measured according to the recently reported protocol $[26,27]$. The sample $(20 \mu \mathrm{L})$ was pipetted to the proper well and incubated for $10 \mathrm{~min}$ with fluorescein as the fluorescent probe. The final assay solution contained 
$6.7 \times 10^{-8} \mathrm{~mol} \cdot \mathrm{L}^{-1}$ of fluorescein, $6.0 \times 10^{-2} \mathrm{~mol} \cdot \mathrm{L}^{-1}$ of $2,2^{\prime}-$ azobis-2-methyl-propanimidamide, $300 \mu \mathrm{L}$ of sample, or $7 \%$ $\beta$-cyclodextrin as a reagent blank. The fluorescence of an assay mixture was measured and recorded once every minute. The trolox equivalent (TE) was calculated using a standard curve prepared with trolox and used to compare the ORAC of various samples via expression as mol. $\mathrm{kg}^{-1} \mathrm{TE}$.

2.8. Aroma Comparison. Aroma of the samples was compared via an electronic nose PEN3 (Airsense Analytics $\mathrm{GmbH}$, Schwerin, Germany). The electronic nose was turned on for $30 \mathrm{~min}$ and was cleaned by flushing the testing system with filtered air at a flow rate of $400 \mathrm{~mL} \cdot \mathrm{min}^{-1}$ for $3 \mathrm{~min}$. The electronic sensor was immediately inserted to the testing tube after the sample of $2 \mathrm{~mL}$ was put into the tube at $25^{\circ} \mathrm{C}$ for $30 \mathrm{~min}$. The responses of the sensor were collected for $2 \mathrm{~min}$ with an interval of $1 \mathrm{~s}$. During the data collecting, the chamber flow was $400 \mathrm{~mL} \cdot \mathrm{min}^{-1}$ with filtered air. The resulting data were further analyzed by principal component analysis (PEN3 and WinMuster, Version 1.6.2, Airsense Analytics GmbH, Schwerin, Germany).

2.9. Statistical Analysis. The experiment was repeated 3 times. The data were expressed as mean \pm standard deviation of at least nine repetitions. Analysis of variance was used to compare mean differences of the results. If differences among means were detected, multiple comparisons were performed using Duncan's Multiple Range Test. Directional difference tests were employed to enable direct comparison of the results of the sensory evaluations. All analyses were conducted in SPSS (Windows Version 19).

\section{Results and Discussion}

3.1. Effect of Light Exposures on Appearance of Fresh-Cut Watermelon. The effect of light exposure on the color of the watermelon is listed in Table 1. Usually, the color can be discriminated when the color difference $(\Delta E)$ was higher than 3.0 [28]. The $\Delta E$ of the blue exposure and the control was 3.36 and 5.00, respectively, while that of the red and green exposures was 0.58 and 2.47 , respectively. The $\Delta E$ of the red exposure was the smallest among all tested light exposures, while that of the control was the largest. Specifically, the $a$ value of the red and green exposures was statistically similar to that of the fresh but was significantly higher than that of the control and blue exposure. The red and green exposures were better to maintain the redness of the fresh-cut watermelon. Hence, the red exposure maintained the original color of the fruit as well as its redness. Similar to our results, the red exposure maintains the original color of the broccoli [14], while the white exposure leads to a significant difference in $a$ and $b$ values of fresh-cut apples [9]. On the other hand, the intense white light pulse exposure of $1.2 \times 105 \mathrm{~J} \cdot \mathrm{m}^{-2}$ leads the redness of the watermelon surface to orange chromaticity [18]. Consequently, both the appropriate spectrum and intensity were key to maintain the original color of the fresh-cut watermelon.
The water-soaking symptom of watermelons is characterized as tissue translucency, softening, and maceration of the endocarp and placental tissues [3, 4]. A number of factors are suspected to contribute to this symptom [3, 29-32].The water-soaking symptom of the fresh-cut watermelon is shown in Figure 2. The water-soaking symptom spreads from the edge of the fruit cube to the whole cube, being consistent with our recently reported manuscript [8]. The water-soaking ratio of the control, red, green, and blue exposures was $28.2 \%, 10.5 \%, 9.2 \%$, and $21.1 \%$, respectively. The water-soaking ratio of the red and green exposures was reduced by $62.8 \%$ and $67.4 \%$ of the control, respectively. Hence, the red and green exposures delayed the water-soaking symptom of the fresh-cut watermelon.

The effect of light exposures on the firmness of fresh-cut watermelon is shown in Figure 3. The firmness of the red and green exposures was significantly higher than that of the control, while that of the blue exposure was similar to that of the control. This phenomenon further validated that the firmness is negatively related to the water-soaking symptom of the watermelon [4]. Being different from our results, the blue exposure maintains the firmness of tomato, whose firmness is higher than that of the red exposure [33]. This difference could result from the higher sensitivity of the tomato epidermis to blue light. Based on the results of the color, water-soaking symptom, and firmness, red light exposure was better to maintain the appearance of the freshcut watermelon.

\subsection{Effect of Light Exposures on the Quality of Fresh-Cut} Watermelon. Figure 4 shows the effect of light exposures on the weight loss, Vc content, soluble solid content, and titratable acid of fresh-cut watermelon. The weight loss of the red and green exposures was significantly lower than that of the white exposure, while that of the blue exposure was higher than that of the white exposure. The weight losses of the red and green exposures were $1.81 \%$ and $2.92 \%$, respectively, which were reduced by $51.1 \%$ and $21.1 \%$ of that of the white light exposure. Consistent with our results, the weight loss of cowpea exposed to red and green light is significantly lower than that of white light [34]. Moreover, the weight loss of the fruit and vegetable is positively linearly related with light intensity [8]. For instance, the weight loss of lettuce is $1.74 \%, 1.35 \%$, and $1.05 \%$ for exposures of 2500 lux, 500 lux, and dark condition, respectively [16], and that of cauliflower heads is $1.8 \%$ and $1.4 \%$ for exposures of $2.4 \times 10^{-5} \mathrm{~mol} \cdot \mathrm{m}^{-2} \cdot \mathrm{s}^{-1}$ and dark conditions, respectively [11]. Consequently, the light intensity was another important factor influencing the weight loss of the sample.

The soluble solid content of each exposure was significantly enhanced compared to that of the control. This phenomenon mainly resulted from the weight loss during the retail display and the sugar metabolism of fruits [35]. Remarkably, the red and green exposures raised the soluble solid content significantly compared to the white exposure, while the blue exposure did not. Consistent with our results, 
TABLE 1: Effect of light exposures on color of the fresh-cut watermelon.

\begin{tabular}{|c|c|c|c|c|c|c|c|}
\hline & $L_{1}$ & $L_{0}$ & $a_{1}$ & $a_{0}$ & $b_{1}$ & $b_{0}$ & $\Delta E$ \\
\hline Control & $34.06 \pm 0.17 a^{*}$ & $30.02 \pm 0.09$ & $7.21 \pm 0.14 b^{*}$ & $8.45 \pm 0.47$ & $6.86 \pm 0.23 a^{*}$ & $4.18 \pm 0.36$ & 5.00 \\
\hline Red & $29.62 \pm 0.11^{c}$ & $30.02 \pm 0.09$ & $8.38 \pm 0.18^{\mathrm{a}}$ & $8.45 \pm 0.47$ & $3.76 \pm 0.11 \mathrm{~d} a^{*}$ & $4.18 \pm 0.36$ & 0.58 \\
\hline Green & $32.32 \pm 0.15 b^{*}$ & $30.02 \pm 0.09$ & $8.71 \pm 0.12^{\mathrm{a}}$ & $8.45 \pm 0.47$ & $5.05 \pm 0.08 b^{*}$ & $4.18 \pm 0.36$ & 2.47 \\
\hline Blue & $32.72 \pm 0.18 \mathrm{ab}^{*}$ & $30.02 \pm 0.09$ & $7.36 \pm 0.08 b^{*}$ & $8.45 \pm 0.47$ & $5.86 \pm 0.13 \mathrm{ca} a^{*}$ & $4.18 \pm 0.36$ & 3.36 \\
\hline
\end{tabular}

Data are means \pm standard deviation $(n \geq 3)$. Data with an asterisk as superscript represent a significant difference with the corresponding initial value $(P<0.05)$. Data with a different letter represent a significant difference in the same column $(P<0.05)$.

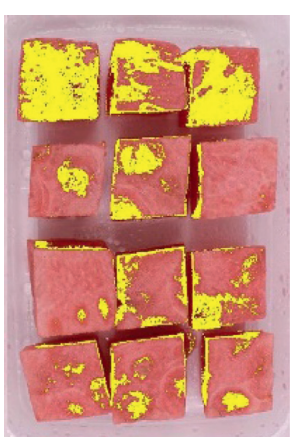

(a)

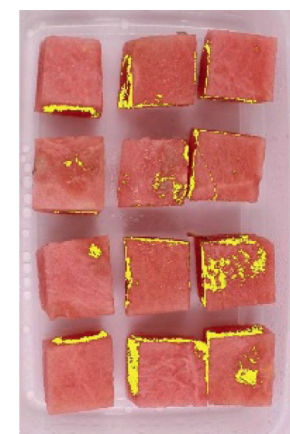

(b)

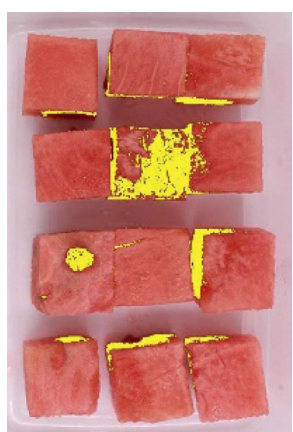

(c)

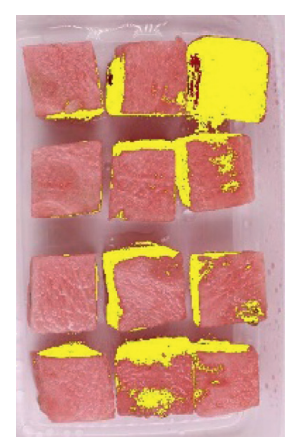

(d)

FIGURE 2: Effect of light exposures on water-soaking symptom of the fresh-cut watermelon. The water-soaking symptom is marked in yellow. Data are mean \pm standard deviation $(n \geq 3)$. Means with different letter represent a significant difference $(P<0.05)$ : (a) control, (b) red, (c) green, and (d) blue.

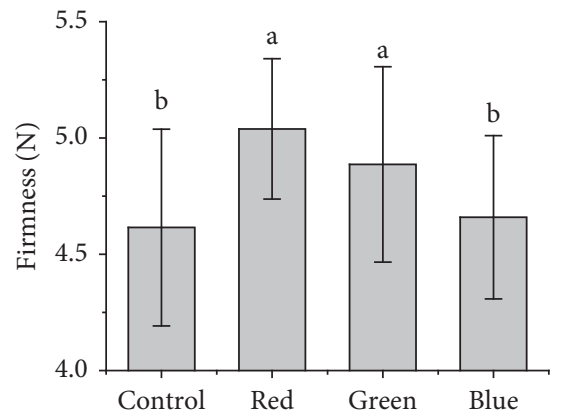

Figure 3: Effect of light exposures on firmness of the fresh-cut watermelon. Data are mean \pm standard deviation $(n \geq 3)$. Means with different letter represent a significant difference $(P<0.05)$.

the soluble solid content of the fresh-cut romaine lettuce shows similar trends after a light exposure of 2500 lux [15].

The titratable acid of the fresh-cut watermelon was significantly enhanced after each light exposure. The green light exposure increased the titratable acid of the fresh-cut watermelon significantly when compared to white light exposure, while the red and blue light exposures did not. Similar to the increase of the soluble solid content, the increase of titratable acid content was mainly related to the respiration of fruits. A lower temperature during the retail display inhibits the respiration, thus maintaining the content of the titratable acid [36].

$\mathrm{Vc}$ is generally regarded as an abiotic stress product of plants. Any stress responses usually lead to an increase of the Vc content followed by a decrease [37], which has been validated during the storage of romaine lettuce and broccoli during the white exposure of 2500 lux $[10,15]$. Each exposure reduced the $\mathrm{Vc}$ content of watermelon cubes. The residual of the $\mathrm{Vc}$ content was as follows: white $>^{\circ}$ blue $^{\circ}>{ }^{\circ}$ green $^{\circ}>{ }^{\circ}$ red. Hence, red and green light exposures were adverse to maintain the $\mathrm{Vc}$ content of fruit cubes. Moreover, the reduction of the $\mathrm{Vc}$ content is related to the light intensity and cultivars. For instance, the reduction of the Vc content of the cauliflower head is positively related to the light intensity of $2.4 \times 10^{-5} \mathrm{~mol} \cdot \mathrm{m}^{-2} \cdot \mathrm{s}^{-1}$ and dark conditions [11]. The reduction of the Vc content is $\leq 5 \%$ for fresh-cut mango, strawberry, and watermelon cubes after six days at $5^{\circ} \mathrm{C}, 10 \%$ in fresh-cut pineapple pieces, $12 \%$ in fresh-cut kiwifruit slices, and $25 \%$ in fresh-cut cantaloupe cubes [38]. 


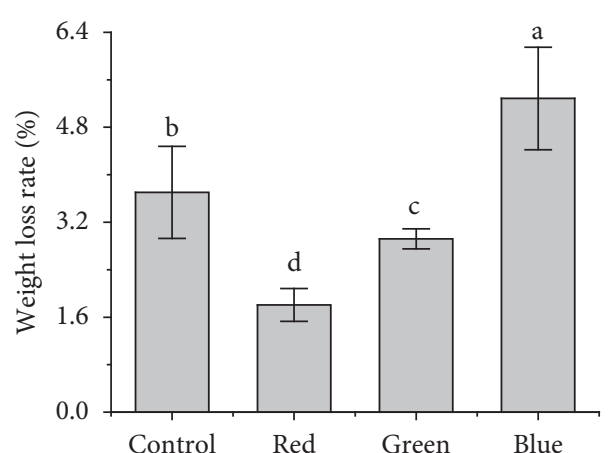

(a)

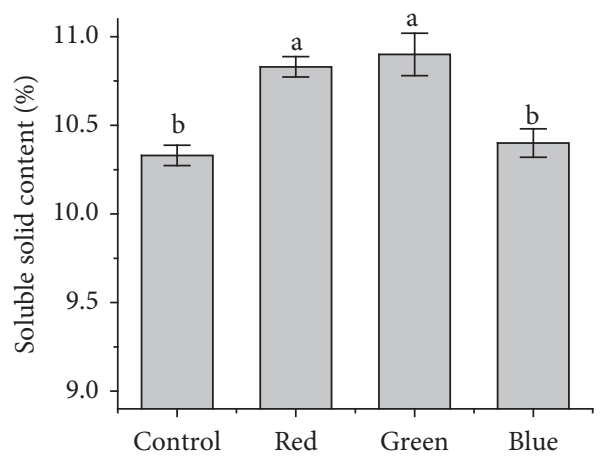

(c)

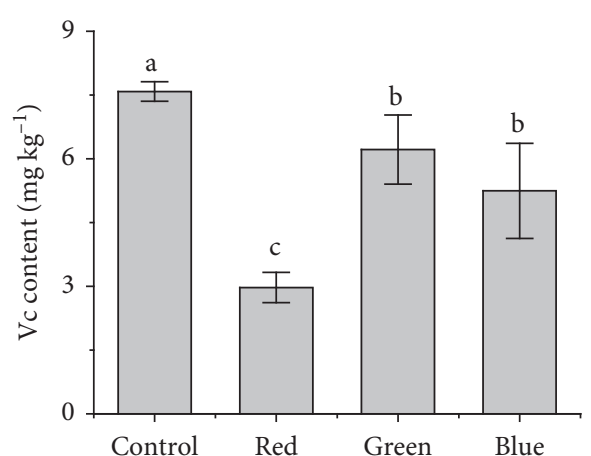

(b)

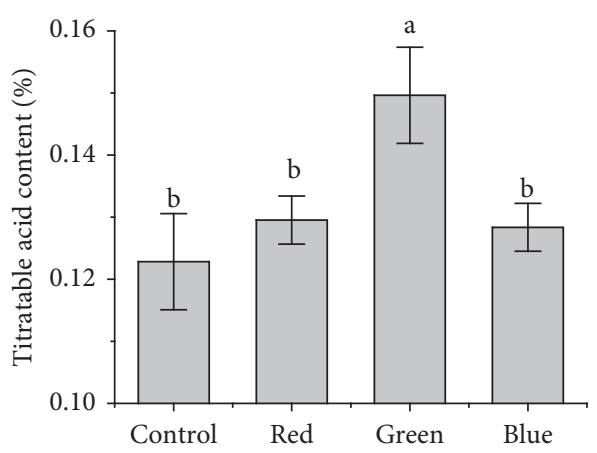

(d)

Figure 4: Effect of light exposures on weight loss (a), Vc content (b), soluble solid content (c), and titratable acid (d) of fresh-cut watermelon. Data are mean \pm standard deviation $(n \geq 3)$. Means with different letter represent a significant difference $(P<0.05)$.

\subsection{Effect of Light Exposure on Antioxidant Capacities of} Fresh-Cut Watermelon. Antioxidant capacity is a further biochemical index of cell metabolism correlated with oxidative stress, which was evaluated via ORAC and DPPH radical methods. The ORAC value reflects the scavenging capacity of a sample to radicals produced during the cell metabolism which mainly transfers labile $\mathrm{H}$ atoms in a lipophilic media, while DPPH radical assesses the ability of the hydroxyl compound to transfer labile $\mathrm{H}$ atoms to the radical mainly in a hydrophilic media [25]. The effect of light exposures on antioxidant capacities of fresh-cut watermelon is shown in Figure 5. Each exposure significantly decreased the antioxidant capacities of the fruit cubes. Moreover, the ORAC value and DPPH radical scavenging capacity were similar as follows: white $>$ blue $>$ green $>$ red, which showed the same order of residual of the $\mathrm{Vc}$ content. The white exposure was better to maintain the antioxidant capacities of the fresh-cut watermelon than the other exposures. Remarkably, the ORAC value of the white exposure was reduced by $18.9 \%$, while the DPPH radical scavenging capacity was reduced by $45.3 \%$. The reduction of the ORAC value was smaller than that of the DPPH radical scavenging capacity. The antioxidant capacities to the radicals produced during the cell metabolism were better to be retained. Therefore, the biological radical scavenging capacity was well maintained by each light exposure although the antioxidant capacities of both biological and chemical radicals were reduced.
Moreover, the antioxidant capacities of the exposed and unexposed fruit cubes were compared. The antioxidant capacity of exposed cubes was significantly lower than that of unexposed cubes. The antioxidant capacity of fruit cubes was negatively related to the exposure intensity. Hence, each light exposure led to a reduction of antioxidant capacities of the fresh-cut watermelon.

3.4. Effect of Light Exposures on Aroma of Fresh-Cut Watermelon. The aroma of the fresh-cut watermelon was compared via a sensor array of 10 electrodes of an electric nose. The dimensions of the electrical response (10 groups) were reduced via principal component analysis. The main components 1 and 2 contributed $98.5 \%$ and $0.97 \%$ of the total watermelon aroma, respectively (Figure 6). The total variance of the main components 1 and 2 reached $99.6 \%$. Consequently, the main components 1 and 2 were used to discriminate the aroma difference of the light exposures. Specifically, the aroma of the red exposure overlapped with that of the control based on the main component 1 , while the white, blue, and green exposures did not. The aroma of each treatment was similar to that of the control based on main component 2 . Hence, red light exposure delayed the aroma deterioration of fresh-cut watermelon.

The loading analysis presented the contribution of each electrode to the main components 1 and 2. Each electrode added a positive contribution to the main component 1 with 


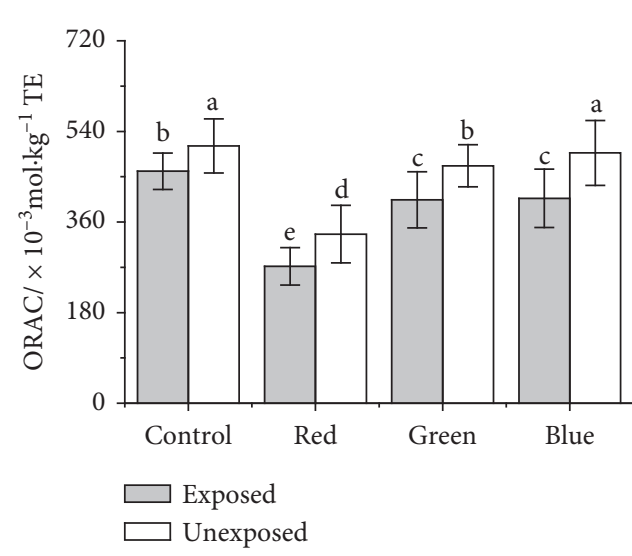

(a)

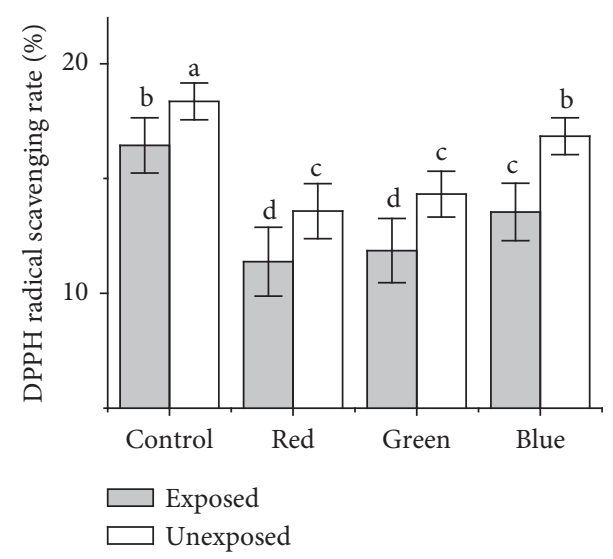

(b)

FIGURE 5: Effect of light exposures on ORAC value (a) and DPPH radical scavenging capacity (b) of fresh-cut watermelon. Data are mean \pm standard deviation $(n \geq 3)$. Means with different letter represent a significant difference $(P<0.05)$.

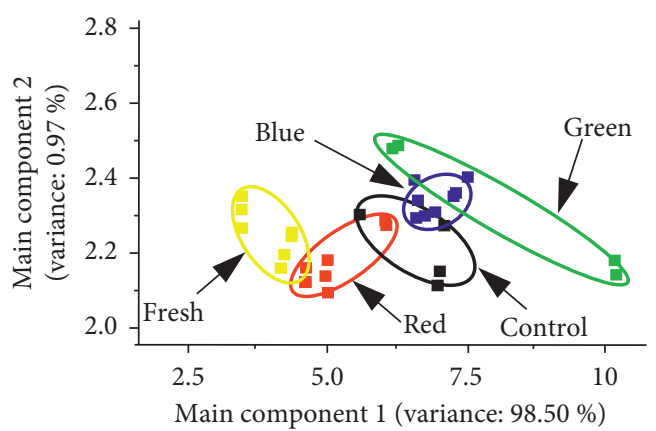

(a)

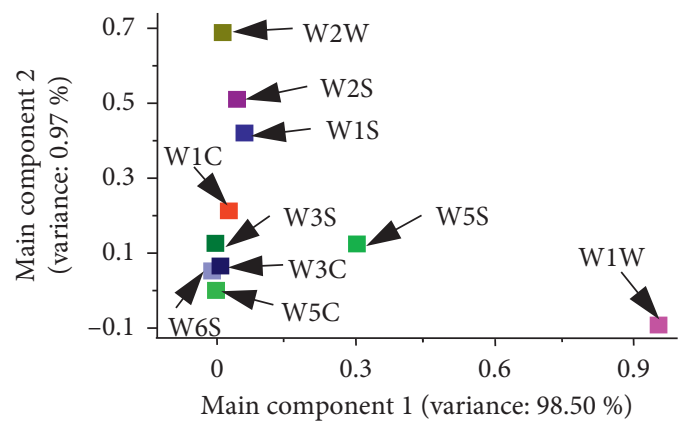

(b)

FIGURE 6: (a) Effect of light exposures on aroma of fresh-cut watermelon and (b) contribution of the electrode to the principal components.

W2S, W5S, and W1S adding the main contributions. Each electrode, except for $\mathrm{W} 1 \mathrm{~W}$, added a positive contribution to the main component 2 with W2W and W2S adding the main contributions. The variances of the main components 1 and 2 were $98.5 \%$ and $0.97 \%$, respectively. Consequently, W1W and W5S sensors were the main basis for judging the aroma change of fresh-cut watermelon. These phenomena could be the result of a small amount of sulfide metabolized by microbes during the retail display due to the sensitivity of the W1W sensor to inorganic sulphides (Pen3). Moreover, the main volatile components of watermelon are nonenol/ nonenal and their derivatives due to the sensitivity of the W5S sensor to the short or aromatic components of alkanes (Pen3). Hence, the aroma change could be the result of microbial metabolism and the self-metabolism of the volatiles of fresh-cut watermelon.

\section{Conclusion}

The fresh-cut watermelons were exposed to the red, green, blue, and white light at $4^{\circ} \mathrm{C}$ for four days, respectively. The maximum emissions of the red, green, blue, and white lights were located in 620-650, 510-530, 440-460, and 430-460 and 500-640 nm, respectively. The weight loss of the red and green light exposures was $1.81 \%$ and $2.92 \%$, respectively, which was reduced by $51.1 \%$ and $21.1 \%$ of the white light exposure. The color difference of the red light exposure was the smallest among all the light exposure, while that of the white light exposure was the largest. The red and green exposures were better to maintain the original color of fresh-cut watermelon as well as their redness. Meanwhile, the red and green exposures delayed the water-soaking symptom and maintained the firmness of the fruit cubes. Moreover, the aroma deterioration was delayed by the red light exposure, which resulted from microbial metabolism and the self-metabolism of volatiles of the fresh-cut watermelon. However, each light exposure significantly decreased the antioxidant capacities of the fruits. The ORAC value and DPPH radical scavenging capacity were similar as follows: white $>$ blue $>$ green $>$ red. In summary, red light exposure could be a better option to delay the appearance and aroma deterioration of fresh-cut watermelons during their retail display.

\section{Data Availability}

The data used to support the findings of this study are available from the corresponding author upon request. 


\section{Conflicts of Interest}

The authors declare that they have no conflicts of interest.

\section{Acknowledgments}

The authors are grateful to financial support of the China Agricultural Research System (CARS-25), Collaborative Innovation Center of the Beijing Academy of Agricultural and Forestry Sciences (KJCX201915), and the (QNJJ201802).

\section{References}

[1] W. G. McGlynn, D. D. Bellmer, and S. S. Reilly, "Effect of precut sanitizing dip and water jet cutting on quality and shelf-life of fresh-cut watermelon," Journal of Food Quality, vol. 26, no. 6, pp. 489-498, 2003.

[2] P. Petrou, G. Soteriou, R. E. Schouten, and M. C. Kyriacou, "Effects of rind removal on physicochemical quality characteristics of fresh-cut watermelon Citrullus lanatus (Thunb) Matsum \& Nakai during cold storage," International Journal of Food Science \& Technology, vol. 48, no. 2, pp. 357-362, 2013.

[3] L. Mao, Y. Karakurt, and D. J. Huber, "Incidence of watersoaking and phospholipid catabolism in ripe watermelon (Citrullus lanatus) fruit: induction by ethylene and prophylactic effects of 1-methylcyclopropene," Postharvest Biology and Technology, vol. 33, no. 1, pp. 1-9, 2004.

[4] C. Zhang, L. I. W. Huo Jie, Y. Ma, G. Gong, X. Zhao, and $\mathrm{Y}$. Xu, "Preharvest treatment of 1-MCP reduces the occurrence of the water-soaking and enhances the quality of watermelons," Ausing Journal of Nutrition and Food Sciences, vol. 3, pp. 1-4, 2015.

[5] I. Aguiló-Aguayo, M. Montero-Calderón, R. Soliva-Fortuny, and O. Martín-Belloso, "Changes on flavor compounds throughout cold storage of watermelon juice processed by high-intensity pulsed electric fields or heat," Journal of Food Engineering, vol. 100, no. 1, pp. 43-49, 2010.

[6] X. Tang, H. He, Y. Liu, and X. Zhao, "Identification of aroma compounds in watermelon juice by SPME-GCMS," Acta Horticulturae, vol. 944, pp. 183-191, 2012.

[7] Y. Wang, W. Li, Y. Ma, X. Zhao, and C. Zhang, "Effect of thermal treatments on quality and aroma of watermelon juice," Journal of Food Quality, vol. 2018, Article ID 9242675 , 10 pages, 2018.

[8] Y. Wang, W. Li, W. Cai et al., "Visible light exposure reduces the drip loss of fresh-cut watermelon," Journal of Food Science and Technology, vol. 55, no. 5, pp. 1816-1822, 2018.

[9] L. Manzocco, B. Quarta, and A. Dri, "Polyphenoloxidase inactivation by light exposure in model systems and apple derivatives," Innovative Food Science \& Emerging Technologies, vol. 10, no. 4, pp. 506-511, 2009.

[10] L. Zhan, J. Hu, Y. Li, and L. Pang, "Combination of light exposure and low temperature in preserving quality and extending shelf-life of fresh-cut broccoli (Brassica oleracea L.)," Postharvest Biology and Technology, vol. 72, pp. 76-81, 2012.

[11] L. Zhan, J. Hu, L. Pang, Y. Li, and J. Shao, "Light exposure reduced browning enzyme activity and accumulated total phenols in cauliflower heads during cool storage," Postharvest Biology and Technology, vol. 88, pp. 17-20, 2014.

[12] E. Artés, E. Petrussa, C. Peresson et al., "Low-intensity light cycles improve the quality of lamb's lettuce (Valerianella olitoria L. Pollich) during storage at low temperature," Postharvest Biology and Technology, vol. 90, pp. 15-23, 2014.
[13] M. J. Kim, C. H. Tang, W. S. Bang, and H. G. Yuk, "Antibacterial effect of $405 \pm 5 \mathrm{~nm}$ light emitting diode illumination against Escherichia coli O157:H7, Listeria monocytogenes, and Salmonella on the surface of fresh-cut mango and its influence on fruit quality," International Journal of Food Microbiology, vol. 244, pp. 82-89, 2017.

[14] M. J. Kim, W. S. Bang, and H. G. Yuk, " $405 \pm 5$ nm light emitting diode illumination causes photodynamic inactivation of Salmonella spp. on fresh-cut papaya without deterioration," Food Microbiology, vol. 62, pp. 124-132, 2017.

[15] L. Zhan, J. Hu, Z. Ai, L. Pang, Y. Li, and M. Zhu, "Light exposure during storage preserving soluble sugar and l-ascorbic acid content of minimally processed romaine lettuce (Lactuca sativa L.var. Longifolia)," Food Chemistry, vol. 136, no. 1, pp. 273-278, 2013.

[16] L. Zhan, Y. Li, J. Hu, L. Pang, and H. Fan, "Browning inhibition and quality preservation of fresh-cut romaine lettuce exposed to high intensity light," Innovative Food Science \& Emerging Technologies, vol. 14, pp. 70-76, 2012.

[17] F. Charles, P. Nilprapruck, D. Roux, and H. Sallanon, "Visible light as a new tool to maintain fresh-cut lettuce post-harvest quality," Postharvest Biology and Technology, vol. 135, pp. 51-56, 2018.

[18] A. Y. Ramos-Villarroel, N. Aron-Maftei, O. Martín-Belloso, and R. Soliva-Fortuny, "Influence of spectral distribution on bacterial inactivation and quality changes of fresh-cut watermelon treated with intense light pulses," Postharvest Biology and Technology, vol. 69, pp. 32-39, 2012.

[19] F. Artés-Hernández, P. A. Robles, P. A. Gómez, A. T. Callejas, and F. Artés, "UV-C illumination for keeping overall quality of fresh-cut watermelon," Postharvest Biology and Technology, vol. 55, no. 2, pp. 114-120, 2009.

[20] W. Cai, W. Li, Y. Li, Y. Ma, X. Zhao, and C. Zhang, "Screening for fresh-cut watermelon from selected cultivars," in Proceedings of International Conference on Computer Science and Environmental Engineering, pp. 155-160, DEStech Publications, Inc., Beijing, China, May 2015.

[21] J. M. Fonseca and J. W. Rushing, "Effect of ultraviolet-C light on quality and microbial population of fresh-cut watermelon," Postharvest Biology and Technology, vol. 40, no. 3, pp. 256-261, 2006.

[22] L. Mao, J. Jeong, F. Que, and D. J. Huber, "Physiological properties of fresh-cut watermelon (Citrullus lanatus) in response to 1-methylcyclopropene and post-processing calcium application," Journal of the Science of Food and Agriculture, vol. 86, no. 1, pp. 46-53, 2006.

[23] S. Robert, Y. Luo, and J. L. McEvoy, "Quality characteristics of fresh-cut watermelon slices from non-treated and 1-methylcyclopropene-and/or ethylene-treated whole fruit," Postharvest Biology and Technology, vol. 44, pp. 71-79, 2007.

[24] G. Oms-Oliu, I. Odriozola-Serrano, R. Soliva-Fortuny, and O. Martín-Belloso, "Effects of high-intensity pulsed electric field processing conditions on lycopene, vitamin $\mathrm{C}$ and antioxidant capacity of watermelon juice," Food Chemistry, vol. 115, no. 4, pp. 1312-1319, 2009.

[25] D. Villaño, M. S. Fernández-Pachón, M. L. Moyá, A. M. Troncoso, and M. C. García-Parrilla, "Radical scavenging ability of polyphenolic compounds towards DPPH free radical," Talanta, vol. 71, no. 1, pp. 230-235, 2007.

[26] B. Ou, D. Huang, M. Hampsch-Woodill, J. A. Flanagan, and E. K. Deemer, "Analysis of antioxidant activities of common vegetables employing oxygen radical absorbance capacity (ORAC) and ferric reducing antioxidant power (FRAP) 
assays: a comparative study," Journal of Agricultural and Food Chemistry, vol. 50, no. 11, pp. 3122-3128, 2002.

[27] K. Zhou and L. Yu, "Effects of extraction solvent on wheat bran antioxidant activity estimation," LWT-Food Science and Technology, vol. 37, no. 7, pp. 717-721, 2004.

[28] I. Kučerová, S. Marek, and J. Banout, "Solar drying and sensory attributes of eland (Taurotragus oryx) jerky," Journal of Food Quality, vol. 2018, Article ID 1067672, 10 pages, 2018.

[29] M. Cho, B. M. Hurr, J. Jeong, C. Lim, and D. J. Huber, "Postharvest senescence and deterioration of "Thoroughbred" and "Carlo" green beans (Phaseolus vulgaris L.) in response to 1-methylcyclopropene," HortScience, vol. 43, no. 2, pp. 427-430, 2008.

[30] B. M. Hurr, D. J. Huber, C. E. Vallejos, E. Lee, and S. A. Sargent, "Ethylene-induced overproduction of reactive oxygen species is responsible for the development of watersoaking in immature cucumber fruit," Journal of Plant Physiology, vol. 170, no. 1, pp. 56-62, 2013.

[31] S. G. Lee and K. D. Ko, "Ethephon application induces symptoms of fruit tissue degeneration in watermelon," Journal of Plant Biology, vol. 51, no. 5, pp. 337-340, 2008.

[32] S. G. Lee, Y. A. Shin, K. Y. Kim, J. H. Chung, and Y. B. Lee, "Effect of rootstocks on the growth, fruit quality and ethylene evolution from harvested fruits in watermelon," Journal of the Korean Society for Horticultural Science, vol. 39, pp. 238-241, 1998.

[33] R. Dhakal and K. H. Baek, "Short period irradiation of single blue wavelength light extends the storage period of mature green tomatoes," Postharvest Biology and Technology, vol. 90, pp. 73-77, 2014.

[34] Y. Shi, Q. Zheng, Q. Wang, L. P. Gao, and J. H. Zuo, "Effect of LED exposure on postharvest qualities of cowpea," Science and Technology of Food Industry, vol. 39, pp. 46-52, 2018, in Chinese.

[35] T. Hou, S. Song, W. Li, W. Wang, and S. Zheng, "Effect of cultivars on qualities of watermelon during storage at room temperature," Modern Food Technology, vol. 27, pp. 242-245, 2011, in Chinese.

[36] S. Khalid, A. U. Malik, A. S. Khan et al., "Tree age and fruit size in relation to postharvest respiration and quality changes in "Kinnow" Mandarin fruit under ambient storage," Scientia Horticulturae, vol. 220, pp. 183-192, 2017.

[37] P. Ahmad, C. A. Jaleel, M. A. Salem, G. Nabi, and S. Sharma, "Roles of enzymatic and nonenzymatic antioxidants in plants during abiotic stress," Critical Reviews in Biotechnology, vol. 30, no. 3, pp. 161-175, 2010.

[38] M. I. Gil, E. Aguayo, and A. A. Kader, "Quality changes and nutrient retention in fresh-cut versus whole fruits during storage," Journal of Agricultural and Food Chemistry, vol. 54, no. 12, pp. 4284-4296, 2006.

[39] Mannul of the Pen3. 